\title{
A Stochastic Scheduling Approach for Maintaining Capability Interdependencies and Defence Program Investment
}

\author{
M.-T. Nguyen $^{\mathrm{a}}$ and R. Taylor ${ }^{\mathrm{a}}$ \\ a Defence Science and Technology Organisation, Australia \\ Email: Minh-Tuan.Nguyen@dsto.defence.gov.au
}

\begin{abstract}
Defence decision-makers are faced with the problem of scheduling and allocating funds to sustain identified capability priorities. Subsequently, an integrated Defence Capability Plan (DCP) is then prepared based on these capability development directions. The DCP is a living document influenced by a range of domestic and international factors. It contains projects and sub-projects which have a large number of interdependencies between their milestones. Any change to the DCP or a desire to find potential savings in the early years of the planning horizon brings about a rescheduling requirement. This may be viewed as a complex, multi-criteria decision problem that is amenable to balance of investment mathematical approaches directed at maintaining and observing capability interdependencies.
\end{abstract}

We first assume all inputs in the model are static and unchanging then formulate it as a mixed-integer linear program. Our mathematical programming approach is designed and implemented as a scheduling tool using public software to allow the required calculations to be completely automated.

We then provide management or decision makers flexibility in the optimal decisions by accommodating uncertainty of inputs (if for example at entry to the DCP a funding band might be provided until the project work is initiated or the project enters the Forward Estimates period of the 10 years budget plan) using Monte Carlo simulation together with optimisation. The uncertainty factor can be represented with probability distribution functions (such as Normal, Triangular, etc.) so that a simulation can be run for each trial schedule, then the output of the simulation are applied in the static model and optimal scheduling data computed. By repeating this process over a number of trials we can estimate the mean and variance of key forecast statistics. Instead of obtaining single-point estimates from the optimisation model, we can now obtain a distribution of the decision variables and hence, a range of optimal values for each decision variable. This provides a form of risk analysis in support of decision making.

Finally, a typical analysis and numerical experiment are demonstrated with general guidelines to consider when using the scheduler tool. The analysis using this mathematical optimisation approach with Monte Carlo simulation can be used to present the least disruptive reschedule for various budget levels and a precision level to make decisions about the budget saving.

Keywords: Project scheduling, mathematical programming, Monte-Carlo simulation, decision support tool, Defence capability plan 


\section{INTRODUCTION}

The Australian Government (GovAUS) Department of Defence (DoD) manages over a hundred Defence projects planned to replace, upgrade or supplement the current military force through the Defence Capability Plan (DCP) (Department of Defence, 2012b) and Defence Capability Guide ${ }^{1}$ (DCG) (Department of Defence 2012a). Most Defence projects within the DCP and DCG are in some way interdependent, or connected, to other projects ${ }^{2}$. Examples of interdependencies include the need for several pieces of equipment to work together to serve a combined function, the replacement of one project by another, one project's force-multiplying effect on the effectiveness of other pieces of equipment, or the decision for one project's inclusion into the DCP or DCG being informed by feedback on the operational effectiveness of other projects in service.

Management of the DCP and DCG often involves annual rescheduling of the 'year of approval' of certain projects in response to cost or budget variations. Due to the complexity associated with the various types and number of interdependencies between the schedule milestones of these projects, it is often difficult and time consuming to manually determine (a) feasible, (b) good, or (c) its optimal reschedule. This paper explains how optimal rescheduling options can be computed that take account of uncertainties in input parameters. This can be thought of as a form of risk analysis in support of decision making.

\section{Rescheduling Problem}

\subsection{Project \& Asset Milestones}

As the DCP and DCG are future plans, a project or subproject within them represents an intent, not a commitment, to acquire a new capability, or enhance or replace an existing capability, i.e. a Key Defence Asset (KDA). Following project management best practice, each project or subproject $\left(P_{n}, n \in\{1, \ldots, N\}\right.$, where $N$ is the number of DCP and DCG projects) contains:

- a multi-year estimated funding profile $\left(f_{n}^{t}, n \in\{1, \ldots, N\}\right.$ and $t \in\{1, \ldots, m T\}, T$ is the number of planning years, usually a 10 -year plan $(T=10) ; m$ is a factor so that $m T$ covers the whole funding profile, usually taking a value 2 or 3 ) that the project will incur (monies used to design/build/test or simply purchase), and

- a number of proposed milestones. These include the Year Of Decision $\left(\mathrm{YOD}_{i}\right)$ - the year Government plans to make a final decision on committing to the proposed project, and the In-Service Date $\left(\operatorname{ISD}_{i}\right)$ - the year the project delivers the capability into service.

The difference between $\mathrm{ISD}_{i}$ and $\mathrm{YOD}_{i}$ is the estimated length of time to be spent in the Defence Materiel Organisation (DMO) before realising the capability. Once Project $i$ has reached its $\mathrm{ISD}_{i}$, it becomes a KDA and one of its important milestones is the Planned Withdrawal Date $\left(\mathrm{PWD}_{k}, k \in\{1, \ldots, K\}, K\right.$ is the number of assets) - the year the asset is to be decommissioned.

\subsection{Typical Project Interdependencies}

We restrict attention to the following four types of project interdependencies for our illustration purpose: (1) Replacement (REP) - a project realising a capability that is to replace an existing asset; (2) Requirement (REQ) - a decision on one project must be taken before a decision on another project; (3) Prerequisite (PRE) - a capability realised by one project must be in service before a decision on another project, and (4) Staggering (STA) - a capability realised by one project must be in service before a capability realised by another project. These interdependency conditions can be mathematically presented by

$$
\begin{aligned}
\mathrm{ISD}_{i} & \leq \mathrm{PWD}_{k}, \quad \forall(i, k) \in \mathscr{S}_{\mathrm{REP}}, \\
\mathrm{YOD}_{i} & \leq \mathrm{YOD}_{j}, \quad \forall(i, j) \in \mathscr{S}_{\mathrm{REQ}}, \\
\mathrm{ISD}_{i} & \leq \mathrm{YOD}_{j}, \quad \forall(i, j) \in \mathscr{S}_{\mathrm{PRE}}, \\
\mathrm{ISD}_{i} & \leq \mathrm{ISD}_{j}, \quad \forall(i, j) \in \mathscr{S}_{\mathrm{STA}},
\end{aligned}
$$

where $\mathscr{S}_{p}$ is set of project-project/asset pairs having the interdependency type $p \in\{$ REP, REQ, PRE, STA $\}$.

\footnotetext{
${ }^{1}$ The DCG is a new document and provides general guidance for industry on projects for the six year period following the four years Forward Estimates period of the DCP.

${ }^{2}$ Here we use the word 'project' to broadly cover both a plan for a capability or an in-service capability.
} 


\subsection{Budgetary Guidance and Committee Capacity}

In the Defence context, decision-makers, in particular the Defence Capability Committee (DCC), are faced with the question of which capabilities/projects to invest in to develop the capabilities needed to achieve the government's strategic objectives, subject to budgetary guidance.

The limited resources are the funds available for capability development through the Defence Budget. Some financial guidance is provided for future years but this can be subject to some uncertainty. The competing demands are the capability proposals and projects for developing Australia Defence Force (ADF) capabilities in the future. Particularly, these are the major capital investment projects in the DCP and DCG.

Denote by $b_{t}, t \in\{1, \ldots, T\}$ the budget guidance on the DCP and DCG for the next $T$ years. It is desirable to have

$$
\sum_{t=1}^{T} \sum_{n=1}^{N} f_{n}^{t} \leq \sum_{t=1}^{T} b_{t}
$$

where $T=4$ for the case of the Forward Estimates (FE) years guidance and $T=10$ for the case of the 10-year budget plan. The budget guidance can also be expressed as a percentage variation from the current funding plan. For example, a $5 \%$ saving over the FE, but $10 \%$ increased over the total 10 -year plan.

Also, the workflow of the Defence committee is scheduled to only consider a limit number of projects. Denoting $c_{t}$ as the committee capacity at Year $t, t \in\{1, \ldots, T\}$ we require

$$
\sum_{n=1}^{N} g_{n}^{t} \leq c_{t}, \forall t \in\{1,2, \ldots, T\}, \text { where } g_{n}^{t}= \begin{cases}1, & \text { Project } n \text { having its } \text { YOD }_{n} \text { at Year } t \\ 0, & \text { otherwise. }\end{cases}
$$

Similarly, the capacity of the Defence approval committee is stated as a percentage variation to the current work plan. For example, the capacity of the Defence approval committee in the future years remains at its current level.

\subsection{Critical Capabilities}

Some projects may, for various reasons, be deemed critical, and thus their funding, YOD and ISD must remain unchanged. However, we can not exclude them from the problem as they may have a number of interdependency requirements to other projects. Let $\mathscr{Q}$ represent this quarantine set of projects.

\subsection{The Decision Problem}

It is noted that the DCP and DCG are living documents influenced by a range of domestic and international factors. For example, since the 2009 White Paper (Department of Defence, 2009) a number of significant developments have affected Defence's posture, future force structure and the Defence budget. Consequently, the 2012 Public DCP (Department of Defence, 2012b) contains 111 projects, or phases of projects, worth approximately $\$ 153$ billion in total capital costs. The collection of milestones and their interdependencies (temporal or otherwise) represent a complex and dynamic scheduling challenge, and any change to the current DCP brings about a rescheduling requirement.

A rescheduling may also be initiated by the desire to find potential savings in the early years of the planning horizon. Near term savings can be generated by delaying projects, shifting some or all of the associated costs past the planning horizon. However, this needs to be done with caution as delaying projects reduces the total capability produced during the planning horizon. As the set of DCP projects and subprojects may have a large number of interdependencies, finding an acceptable solution to the rescheduling problem can become a very complex and time consuming task.

Our work in support of this problem consists of developing and applying a mathematical formulation of the rescheduling problem with a decision support tool to help automate some parts of the proces: 3

\section{Mathematical Programming Model and Simulation}

Determining an optimal solution to this funding allocation scheduling problem has features in common with the well-characterised knapsack problem (Winston, 1994, page 468). There are many extensions of this basic

\footnotetext{
${ }^{3}$ Note that the data structure/classification and mathematical model presented in this paper do not represent an agreed or endorsed position by Defence.
} 
knapsack model, e.g. see Brown, Dell and Newman (2004); Greiner et al. (2003); Radulescu and Radulescu (2001) and references therein.

Here the Defence capability interdependency and funding allocation scheduling problem involves minimising the total number of funding changes to the current program investment by varying the YOD (and consequently ISD) of some Defence capability projects, while meeting budget guidance and maintaining the capability interdependency requirements. Our problem has an additional temporal dimension which is not present in the knapsack problem. In the knapsack problem, a resource is selected and used once in a given time period. However, in the funding allocation scheduling problem, the resource has been funded for multiple years. Our model also differs from the above by the nature of the objective functions and the proposed multi-stages analysis. Our previous work on a similar scheduling problem for Defence Logistics Funding (Nguyen and Gill. 2006) also developed a mathematical programming model and decision support tool. The proposed model and project scheduling tool in this work, by contrast differs from the past work by incorporating capability interdependencies and various analysis features within a single program.

The Norwegian Defence Research Establishment developed a stochastic model for this problem by considering the uncertainty of future-year budgets (Fauske, Vestli and Glrum, 2013). We first restrict ourselves here to a deterministic model, with discounted costs to cover an effect on variation of the budget. The data structures for collection and model formulation are simple to implement (Nguyen and Gill, 2014). We then provide management or decision makers flexibility in the optimal decisions by accommodating uncertainty of inputs using Monte Carlo simulation together with optimisation. A decision support tool with model variation, result visualisation and comparison is also developed.

\subsection{Deterministic Model}

Decision Variables: The decision variables of concern can be defined as the indicator of the first investment year for each project. Denote this by $x_{n}^{t}$, where $n \in\{1,2, \ldots, N\}$ is the project number and $t \in\{1,2, \ldots, T\}$ is the number of years out from the current financial year. By definition, it must satisfy the assignment condition:

$$
\sum_{t=1}^{T} x_{n}^{t}=1, \quad \text { where } x_{n}^{t}= \begin{cases}1 & \text { if funding of Project } n \text { is started in year } t \\ 0 & \text { otherwise. }\end{cases}
$$

Let us denote by $s_{n}$ the current first year funded of Project $n$. We then define two pairs of auxiliary variables $\left(r_{n}, l_{n}\right)$ and $\left(c_{n}^{r}, c_{n}^{l}\right)$. The first pair is binary and denoting that Project $n$ has slipped to the right or left from $s_{n}$, respectively; and the second pair is non-negative integer variables counting the number of years that Project $n$ slips to the right or left, respectively. The relation between the decision and auxiliary variables is

$$
\sum_{t=1}^{T} t x_{n}^{t}=s_{n}+c_{n}^{r}-c_{n}^{l}
$$

together with the relation between auxiliary variables itself as

$$
\begin{aligned}
& c_{n}^{l} \leq \min \left\{M\left(1-r_{n}\right), M l_{n}\right\}, \\
& c_{n}^{r} \leq \min \left\{M\left(1-l_{n}\right), M r_{n}\right\},
\end{aligned}
$$

where $M$ is a large number, say $M=10 T$.

Objective Function: Our objective is to minimise the total number of funding changes in the current program investment which can be expressed mathematically by

$$
\min \sum_{n=1}^{N}\left[\phi\left(r_{n}+l_{n}\right)+(1-\phi)\left(c_{n}^{r}+c_{n}^{l}\right)\right],
$$

where $\phi$ is a weighting factor to adjust the relative importance of the number of projects slipped and the total years slipped.

Constraints: The most obvious is a budget constraint:

$$
\sum_{t=1}^{T}\left(1+\rho_{t}\right)^{-t} \sum_{n=1}^{N} \sum_{s=1}^{t} x_{n}^{s} f_{n}^{t-\left(s-s_{n}\right)} \leq\left(1-\beta_{\mathrm{BP}}\right) \sum_{t=1}^{T}\left(1+\rho_{t}\right)^{-t} \sum_{n=1}^{N} f_{n}^{t},
$$

where $\rho_{t}$ is a discount factor to apply to the cost in Year $t$, and $\beta_{\mathrm{BP}}$ is the percentage level of the cut/increased budget guidance. One may wish to include a budget guidance across the 4-year FE period. It can be modelled by the use of 
M.-T. Nguyen \& R. Taylor, A Stochastic Scheduling Approach for Maintaining Capability Interdependencies ...

$$
\sum_{t=1}^{4}\left(1+\rho_{t}\right)^{-t} \sum_{n=1}^{N} \sum_{s=1}^{t} x_{n}^{s} f_{n}^{t-\left(s-s_{n}\right)} \leq\left(1-\beta_{\mathrm{FE}}\right) \sum_{t=1}^{4}\left(1+\rho_{t}\right)^{-t} \sum_{n=1}^{N} f_{n}^{t}
$$

where $\beta_{\mathrm{FE}}$ is the percentage level of the cut/increased forward estimates. Similarly, the Defence approval capacity constraint can be expressed as

$$
\sum_{n=1}^{N} \sum_{s=1}^{t} x_{n}^{s} g_{n}^{t-\left(s-s_{n}\right)} \leq\left(1-\delta_{t}\right) G_{N}^{t}, \forall t \in[1,2, \ldots, T]
$$

where $\delta_{t}$ is the percentage variation to the current capacity $G_{N}^{t}$ at Year $t$ and $G_{N}^{t} \stackrel{\text { def }}{=} \sum_{n=1}^{N} g_{n}^{t}$. Over the years, violations of some of the typical interdependency conditions (described in Section 2.2 may be deemed an 'acceptable risk' by Defence. To relax the corresponding constraint mathematically or to allow the possibility of removing the violation, we define the following indicators for any interdependency type $p \in\{$ REP, REQ, PRE, STA\}:

$$
\alpha_{i j}^{p}= \begin{cases}0, & \text { interdependency between Project/Asset } i \text { and } j \text { is fixed, } \\ 1, & \text { kept at the current situation. }\end{cases}
$$

The four typical interdependency constraints can be now written as

$$
\begin{aligned}
& \sum_{t=1}^{T} t x_{i}^{t}+\left(\mathrm{ISD}_{i}-s_{i}\right) \leq \mathrm{PWD}_{k}+\alpha_{i, k}^{\mathrm{REP}}\left(\mathrm{ISD}_{i}-\mathrm{PWD}_{k}\right), \forall(i, k) \in \mathscr{S}_{\mathrm{REP}}, \\
& \sum_{t=1}^{T} t x_{i}^{t}+\left(\mathrm{YOD}_{i}-s_{i}\right) \leq \sum_{t=1}^{T} t x_{j}^{t}+\left(\mathrm{YOD}_{j}-s_{j}\right)+\alpha_{i, j}^{\mathrm{REQ}}\left(\mathrm{YOD}_{i}-\mathrm{YOD}_{j}\right), \forall(i, j) \in \mathscr{S}_{\mathrm{REQ}}, \\
& \sum_{t=1}^{T} t x_{i}^{t}+\left(\mathrm{ISD}_{i}-s_{i}\right) \leq \sum_{t=1}^{T} t x_{i}^{t}+\left(\mathrm{ISD}_{j}-s_{j}\right)+\alpha_{i, j}^{\mathrm{PRE}}\left(\mathrm{ISD}_{i}-\mathrm{ISD}_{j}\right), \forall(i, j) \in \mathscr{S}_{\mathrm{PRE}}, \\
& \sum_{t=1}^{T} t x_{i}^{t}+\left(\mathrm{ISD}_{i}-s_{i}\right) \leq \sum_{t=1}^{T} t x_{j}^{t}+\left(\mathrm{YOD}_{j}-s_{j}\right)+\alpha_{i, j}^{\mathrm{STA}}\left(\mathrm{ISD}_{i}-\mathrm{YOD}_{j}\right), \forall(i, j) \in \mathscr{I}_{\mathrm{STA}}, \\
& i, j \in[1, \ldots, N] ; i \neq j ; k \in[1, \ldots, K] .
\end{aligned}
$$

The final constraint is to ensure the projects in the quarantine set $\mathscr{Q}$ (Section 2.4) are excluded from the decision variables

$$
x_{n}^{s_{n}}=1, \quad \forall n \in \mathscr{Q} .
$$

To allow for control of the maximum number of years slipped to the right for each project, we introduce the parameter $C_{n}^{r}$ and express the condition as

$$
\sum_{t=1}^{T} t x_{n}^{t}-s_{n} \leq C_{n}^{r}, \quad \forall n
$$

To control the maximum number of project slipped right, we introduce the parameter $R^{\max }$ such that

$$
\sum_{n=1}^{N} r_{n} \leq R^{\max }
$$

Note that one may wish to find the maximum funding savings from the budget while maintaining all interdependency condition and critical requirements. That means treating the left hand side of (6) or (7) as our objective function, while all other constraints (1)-(4) and (8)-(15) remain.

\subsection{Monte Carlo Simulation}

The model above is not set up to handle "uncontrolled" uncertainty, and requires static values for any factor that is not being adjusted by the optimisation. For example at entry to the DCP a funding band might be provided until the project work is initiated or the project enters the Forward Estimates period of the 10 years budget plan (Capability Development Group, 2014). Here, the uncertain project funding/cost can be represented with probability distribution functions (such as Normal, Triangular, etc.) so that a Monte Carlo simulation can be run for each trial schedule, then the output of the simulation are applied in the static model and optimal scheduling data computed. By repeating this process over a number of trials we can estimate the mean and variance of key forecast statistics.

Instead of obtaining single-point estimates from the optimisation model, we can now obtain a distribution of the decision variables and hence, a range of optimal values for each decision variable. This provides a form of risk analysis in support of decisions. 
M.-T. Nguyen \& R. Taylor, A Stochastic Scheduling Approach for Maintaining Capability Interdependencies ...

\section{Scheduler Tool Illustrative Example}

In this illustration, we use the same data set published in Nguyen (2014) (96 Projects, 49 assets, 30 interdependency conditions in various types and 46 quarantined projects ${ }^{4}$. By accommodating uncertainty of inputs, we take three project groups $\left(\left\{P_{21}, P_{37}, P_{94}\right\},\left\{P_{38}, P_{48}, P_{56}\right\}\right.$ and $\left.\left\{P_{4}, P_{11}, P_{70}\right\}\right)$ then simulate its funding by three triangular distributions, respectively, using funding bands defined by minimum and maximum values (small funding: $(0,50)$; medium: $(0,100)$; and large: $(0,200))$. The range of outputs of the optimisation process is collected by running 100 trials. To obtain a $95 \%$ confidence interval (CI) of simulated output mean, the above process is repeated 10 times (bootstrap technique). These stochastic features using Monte Carlo simulation have been added into our scheduler tool and its front-end menu is shown in snapshot in Figure 1 .

The class of our mathematical programming (MP) problem is obviously an Integer Linear Program (ILP). For this illustrative example, the model has 1344 variables (1152 of them binary) and 763 constraints. Optimal solutions are produced within a second on an Intel i7 CPU @ $3.4 \mathrm{GHz}$ computer with $8 \mathrm{~GB}$ of RAM using open source solver GLPK (Makhorin, 2012). Therefore, one should expect that the running of simulation experiment above will take at least 15 minutes without using any Parallel Computing (Rosenthal, 2000).

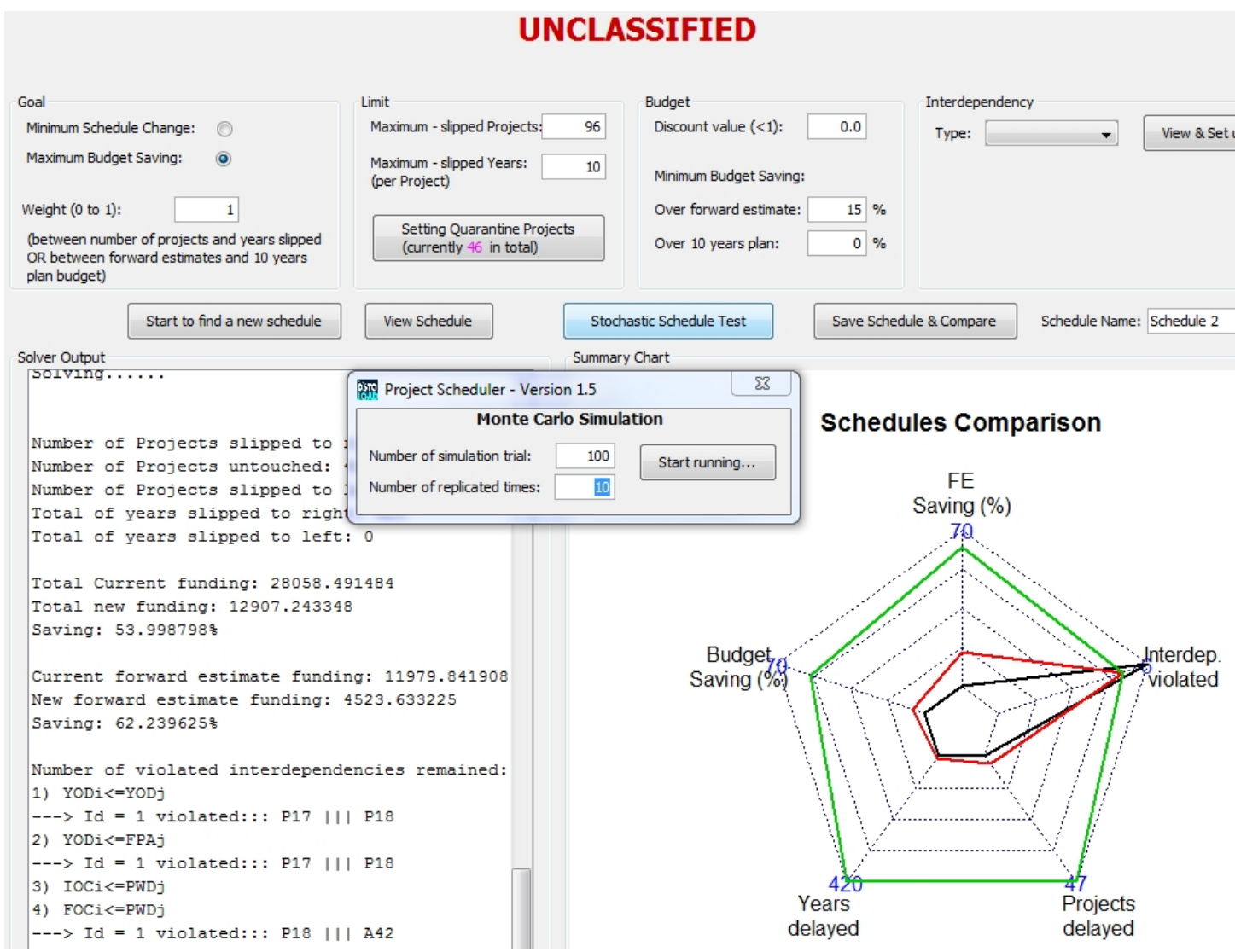

Figure 1: The Scheduler Tool Front-End

Table 1 presents summary results for the illustrative example of some typical analysis using the proposed model and the scheduler tool.

Table 1: Summary of Illustrative Analysis

\begin{tabular}{|l|rr|rr|rr|}
\hline \multirow{2}{*}{ Schedule } & \multicolumn{2}{|c|}{ Max-Save } & \multicolumn{2}{c|}{ Min-Max } & \multicolumn{2}{c|}{ Save-15 } \\
& Range & 95\% CI & Range & 95\% CI & Range & 95\% CI \\
\hline Budget Saving (\%) & $(48.8,50.7)$ & $(50,0,50.2)$ & $(48.0,48.0)$ & $(48.0,48.0)$ & $(5.5,6.4)$ & $(6.0,6.1)$ \\
Forward Estimates Saving (\%) & $(55.6,58.2)$ & $(56.7,56.9)$ & $(52.2,55.2)$ & $(53.3,53.6)$ & $(15.0,16.1)$ & $(15.2,15.3)$ \\
Number of Projects Delayed & $(49,49)$ & $(49,49)$ & $(28,37)$ & $(31,32)$ & $(3,3)$ & $(3,3)$ \\
Number of Years Delayed & $(438,438)$ & $(438,438)$ & $(256,338)$ & $(276,278)$ & $(9,10)$ & $(9,10)$ \\
\hline
\end{tabular}

Max-Save finds the maximum funding saving that can be achieved without violating any constraints,

Min-Max searches for a less disruptive reschedule of the current investment program that achieves almost the maximum saving found above, and

${ }^{4}$ Note that all data presented here is fictitious and used for illustrative purposes only. 
Save- $\beta_{t}$ allows one to obtain other reschedules that meet various levels ( $\beta_{\mathrm{BP}}$ or $\beta_{\mathrm{FE}}$ ) of funding saving with minimum changes.

By applying the Monte Carlo simulation method as explained in Section 3 to this illustrative example we find the budget spend over the planning horizon can be reduced by about $48.8-50.7 \%$ (at the cost of course of increasing spending beyond the planning horizon). However this would involve rescheduling half of all the projects. A similar, but less disruptive option shifts $48 \%$ of the spend by delaying about 28-37 projects. However, if we want to cut $15 \%$ of the FE funding, then only 3 projects need to be delayed. Also the confidence interval is relatively narrow in this illustrative data set. It suggests the robustness of the proposed model and the enough precision to make decisions about the budget saving.

\section{Conclusion}

This paper develops and illustrates a mathematical optimisation approach with Monte Carlo simulation for maintaining capability interdependencies and budgeting program investment. The analysis using this model can be used to present the least disruptive reschedule for various budget levels.

Note that in order to more fully appreciate the value of the proposed model and any case study, the analysis should be compared with the current approach adopted by Defence and the funding solutions it produces. What is clear, however, is that the model and data collection structures assists in making explicit the assumptions, constraints and desired goal as well as providing a tool for automating some of the currently time-consuming manual processes. The formulation of the decision problem as a MP also allows a what-if capability, for example quarantining certain projects from any funding cuts or investigating the impact of fixing some of the current violated interdependencies.

However, the current version of the model is restricted to assuming capability value/benefit of each project as expressed in the constraints and the funding profile is unchanged when moving its first funding year. Also significant verification and validation of the data model are required.

\section{REFERENCES}

Brown, G. G., Dell, R. F. and Newman, A. M. (2004) Optimizing Military Capital Planning, Interfaces 34(6), 415-425.

Capability Development Group (2014) Improving Capability Development: A Discussion Paper. Department of Defence, OCCDG/OUT/2014/69.

Department of Defence (2009) Defence White Paper 2009 - Defence Australia In the Asia Pacific Century: Force 2030, Defence Publishing Service - APR016/08, Australia.

Department of Defence (2012a) Defence Capability Guide 2012- Public Version, Defence Publishing Service - JUL000/12, Australia.

Department of Defence (2012b) Defence Capability Plan 2012- Public Version, Defence Publishing Service MAY015/12, Australia.

Fauske, M., Vestli, M. and Glrum, S. (2013) Optimization Model for Robust Acquisition Decisions in the Norwegian Armed Forces, Interfaces 43(4), 352-359.

Greiner, M. A., Fowler, J. W., Shunk, D. L., Carlyle, W. M. and McNutt, R. T. (2003) A Hybrid Approach Using the Analytic Hierarchy Process and Integer Programming to Screen Weapon Systems Projects, IEEE Transactions on Engineering Management 50(2), 192-202.

Makhorin, A. (2012) GLPK (GNU Linear Programming Kit). http://www.gnu.org/sof tware/glpk/glpk.html

Nguyen, M.-T. (2014) Project Scheduling Tool for Maintaining Capability Interdependencies and Defence Program Investment: A User's Guide. DSTO-GD-0843, Defence Science and Technology Organisation, Australia.

Nguyen, M.-T. and Gill, A. (2006) A Mathematical Programming Approach to Defence Logistics Funding, Australian Society for Operation Research Bulletin 25(6), 2-10.

Nguyen, M.-T. and Gill, A. (2014) An Approach for Maintaining Capability Interdependencies and Budgeting Program Investment, Australian Society for Operation Research Bulletin 33(1), 29-33.

Radulescu, C. Z. and Radulescu, M. (2001) Decision analysis for the project selection problem under risk, in 9th IFAC / IFORS / IMACS / IFIP/Symposium On Large Scale Systems: Theory and Application, Bucharest, pp. 243-248.

Rosenthal, J. S. (2000) Parallel Computing and Monte Carlo Algorithms, Far East Journal of Theoretical Statistics 4(2), 207-236.

Winston, W. L. (1994) Operation Research Applications and Algorithms, Duxbury Press Belmont, CA. 